\title{
NUEVAS VOCES EN CIENCIAS SOCIALES: UNA MIRADA A LA INVESTIGACIÓN SOCIAL
}

\author{
NEW VOICES IN SOCIAL SCIENCES. LOOKING \\ AT THE SOCIAL RESEARCH
}

\author{
En otras palabras, la única razón de ser de nuestras \\ Universidades está el pueblo que las genera, \\ las sostiene y al que ineludiblemente tienen que servir [...] \\ (Baró, 1975: 2) \\ Gloriana Martínez Sánchez ${ }^{1}$ \\ martinez.glory@yahoo.com \\ Adriana Sánchez Lovell ${ }^{2}$ \\ adrilovell@gmail.com
}

\begin{abstract}
Resumen
El presente artículo reflexiona sobre el Encuentro Nuevas Voces en Ciencias Sociales realizado por el Instituto de Investigaciones Sociales de la Universidad de Costa Rica, en el marco de la Semana Universitaria, desde el 2006 hasta la fecha. El artículo contiene tres partes: 1) se presenta al Encuentro, se historiza y se discuten las continuidades, rupturas y transformaciones acaecidas a través de un esfuerzo sostenido de 8 años de trabajo, 2) se caracteriza a Nuevas Voces a la luz de la investigación social: temas, relevancia, aprendizajes y pertinencia de la investigación y 3) cerramos con una reflexión sobre algunos caminos por recorrer, en relación a la producción de conocimiento en ciencias sociales. Palabras claves: Investigación-Ciencias Sociales-Universidad-Sociedad
\end{abstract}

\begin{abstract}
This paper achieves to discuses about students research meeting, "Nuevas Voces en Ciencias Sociales" [New Voices in Social Sciences], by the Institute for Social Research at the University of Costa Rica. In this article, this contains three sections 1) the first one presents the activity and its paths since 2006. 2) Then, discusses its relevance, patters, learning's, and research pertinence. 3) Finally, It closes with a reflection about the production of knowledge in social sciences.
\end{abstract}

Key Words: Research, Social Scienses-University-Society

1 Investigadora del Instituto de Investigaciones Sociales, Universidad de Costa Rica, Sede Rodrigo Facio 2 Investigadora del Instituto de Investigaciones Sociales, Universidad de Costa Rica, Sede Rodrigo Facio 


\section{Introducción}

A partir de la consolidación de las disciplinas de las ciencias sociales en Costa Rica, y su paulatino desarrollo en diversos centros de investigación y casas de estudios superiores -públicas y privadas-, es necesario plantearse las siguientes interrogantes: ¿Qué significa investigar en Ciencias Sociales? y ¿qué implicaciones tiene la labor investigativa en nuestro contexto?

A la luz de las transformaciones socio políticas y los cambios de estilos de desarrollo y de acumulación de capital en el país, el debate sobre el camino de la producción de conocimiento y saberes desde las Ciencias Sociales, así como la relación sociedad-ciencias sociales-universidad toman relevancia.

Según datos oficiales del Consejo Nacional de Rectores (CONARE) existe una gran demanda hacia las carreras de ciencias sociales; por ejemplo, entre los años 2000 y 2007 la mayor cantidad de diplomas otorgados en los centros de educación superior privados y públicos fueron del área de Ciencias Sociales. Asimismo, para el año 2007 las universidades privadas entregaron 8281 diplomas del área de ciencias sociales, y las universidades públicas 3031 diplomas de esta área y 3682 correspondiente al área de educación (Rodríguez, 2008: 13). Estos datos muestran la plena incorporación de los centros de educación superior privados en la formación de las y los profesionales en ciencias sociales, así como la alta demanda que existe para con estas carreras.

Paralelamente a estos procesos, con la profundización de las dinámicas de las políticas neoliberales ${ }^{3}$ la Universidad Pública en América

3 Respecto a la aplicación y profundización del modelo neoliberal en Costa Rica a partir de los años ochenta, Alvarado y Martínez señalan que: “[...] la estrategia neoliberal en Costa Rica ha modificado de modo considerable el escenario económico y social [...] Robles (2011), señala que el proceso de acumulación por desposesión inmerso dentro de la lógica de la estrategia neoliberal encuentra, en el caso costarricense, dos momentos centrales: el del ajuste estructural y el del libre comercio. La remodelación normativa operada bajo el momento del libre comercio implicó la ampliación de nuevos espacios de acumulación por desposesión, los cuales se han colocado en el centro de las luchas sociales y políticas." (2013: 114 y 121).
Latina, entendida históricamente como una institución social que cumple un rol central en la producción de conocimientos y valores-conceptos sociales comunes (Sousa Santos, 2007), se encuentra ante una serie de encrucijadas y contradicciones propias del momento.

Diversas corrientes académicas, donde destacan los análisis de Boaventura de Sousa Santos, señalan que a pesar de la mercantilización- transnacionalización de la educación y la injerencia de organismos de capitales financieros internacionales en las mismas, es posible la construcción de una educación pública de calidad e inclusiva, en servicio de las necesidades e intereses sociales, y no al mando de las directrices de los organismos financieros transnacionales.

De esta manera, a partir del contexto socio-político y las demandas económicas, las disciplinas de las Ciencias Sociales en Costa Rica se mantienen en una constante transformación. En este sentido, algunas unidades académicas de las universidades públicas, no sólo del área de Ciencias Sociales, han emprendido reformas curriculares que incluyen evaluaciones y auditorías externas, en procura de la excelencia académica y de la vinculación de estas carreras con las exigencias del mercado.

Ante estas dinámicas existen tensiones entre diversas posturas que defienden la consolidación de las perspectivas y demandas de la economía neoliberal dentro de las Ciencias Sociales, y perspectivas que plantean rupturas y transformar el status quo de las disciplinas sociales, en pro de una mirada comprometida con los sectores sociales excluidos ${ }^{4}$. La docencia, la investigación, la extensión y acción social se ven atravesadas por estas discusiones, las cuales tienen alcances éticos, políticos y definitorios de las relaciones Universidad y Sociedad. Así lo propuso el conocido psicólogo social y de la liberación, salvadoreño, Martín Baró, cuando en el marco del conflicto armado centroamericano, reflexiona sobre el papel de las universidad pública a mediados de los setentas:

4 Estas tensiones se complejizan aún más cuando se tiene en cuenta las contradicciones que cada una de estas perspectivas encierran, y las tonalidades de grises entre estas posturas. 
"No hay conciencia universitaria donde no hay ciencia; pero una ciencia que pretenda ignorar la realidad histórica en que surge y a la que tiene que responder, una ciencia no comprometida, es una ciencia alienada y alienante" (Martín-Baró, 1975: 640).

De esta manera, tal como lo plantea Martín-Baró, la investigación que se desarrolla desde las Ciencias Sociales define el tipo de universidad que se construye. Es así, que las metodologías, los objetivos y formas de desarrollar y entender la investigación social toman relevancia, ya que determinan el compromiso social, ético y político de la universidad como un bien público, en miras de contribuir al desarrollo y justicia social en su conjunto.

Diversas corrientes teóricas, plantean que a partir de la descolonización y deconstrucción de las lógicas hegemónicas del poder y del conocimiento se abren las puertas para la constitución de universidades públicas accesibles, autónomas, humanistas e inclusivas. Bajo esta misma lógica, aparecen conceptos y propuestas epistemológicas, las cuales plantean la producción de conocimiento a partir del intercambio de saberes diversos, en donde la universidad puede mantener un diálogo constante, horizontal, creativo y reflexivo con diferentes sectores sociales. En relación a lo anterior, Souza Santos expone a la ecología de saberes como una forma para la construcción de conocimientos. Este la define como:

[...] "un conjunto de prácticas que promueven una nueva convivencia activa de saberes con el supuesto de que todos ellos, incluido el saber científico, se pueden enriquecer en ese diálogo [...] sirve de base para la creación de comunidades epistémicos más amplias que convierten a la universidad en un espacio público de inter conocimiento donde los ciudadanos y los grupos sociales pueden intervenir sin la posición exclusiva de aprendices" (Souza Santos, 2007: 33).

Es así, que el papel activo de las y los estudiantes en la creación de saberes -otrosdentro de las estructuras y dinámicas de las universidades es fundamental para un diálogo de saberes. Abrir la mirada de los sectores y/o grupos académicos "más consolidados" a los nuevos investigadores e investigadoras, enriquece la producción de conocimientos, metodologías, y epistemologías que se nutren en sí mismas y que aportan desde -y para- diversos entornos sociales.
Por este motivo, en el presente artículo se recogen las principales experiencias y aprendizajes de la realización de los Encuentro Nuevas Voces en Ciencias Sociales desarrollados anualmente a partir del 2006, entre jóvenes investigadores e investigadoras, en su mayoría estudiantes de la Facultad de Ciencias Sociales, y el Instituto de Investigaciones Sociales (IIS) de la Universidad de Costa Rica (UCR).

De esta manera, se plantea desarrollar a lo largo del artículo las distintas facetas de la producción y organización de los Encuentros Nuevas Voces en Ciencias Sociales, así como las principales enseñanzas y comunes denominadores en relación con la producción de investigación social desde las perspectivas de las y los jóvenes investigadores. Entre estos ejes comunes destacan las nuevas temáticas, diversos abordajes y metodologías, así como algunos retos en relación con la pedagogía de la investigación, reflexiones que aportan a la discusión actual sobre la inter-transdiciplinariedad y las propuestas epistemológicas del diálogo y ecología de saberes.

\section{Nuevas Voces: ¿qué es y cuál es su historia?}

El Encuentro Nuevas Voces en Ciencias Sociales es un esfuerzo que realiza el IIS desde el año 2006, con el fin de mostrar, en el marco de la Semana Universitaria de la UCR, el aporte que realizan las nuevas generaciones de cientistas sociales. Asimismo, Nuevas Voces (NV) busca potenciar los alcances de los Trabajos Finales de Graduación (TFG) de relevancia y pertinencia social producidos en la educación superior pública. ${ }^{5}$

El principal objetivo del Encuentro es cumplir con la esencia de la investigación en la universidad pública -humanista y plural- que basa su acción en los intercambios de ideas y creación de las condiciones necesarias para reflexionar sobre la realidad social. Para las Ciencias Sociales es imprescindible el debate y la apertura hacia

5 Nuevas Voces es posible gracias al apoyo institucional que han brindado las autoridades universitarias a través de los años que tiene de celebrarse el encuentro, en comunión con la organización y participación del personal del Instituto de Investigaciones Sociales. 
la comunidad, como único camino que garantiza su crecimiento y pertinencia, por esta razón NV busca establecer puentes entre las y los jóvenes investigadores, la comunidad universitaria y la sociedad para crear espacios de intercambio de conocimientos, formación de redes, así como la devolución y exposición de los trabajos que realizan las y los investigadores. Carlos Sandoval García, fundador de NV reflexiona a inicios del I Encuentro NV:

"La meta es que las nuevas generaciones de cientistas sociales cuenten con un espacio anual en el cual puedan presentar las principales conclusiones de sus trabajos [...] La Semana Universitaria puede ser una arena ideal para presentar (los). También es nuestro deseo que quienes participen en este Encuentro puedan reconocerse generacionalmente y puedan considerar iniciativas colectivas"6 (Sandoval, 2007).

La modalidad del encuentro de NV consiste en la presentación de una ponencia donde se procura un intercambio abierto. Los trabajos que se presentan pueden estar al inicio del proceso de investigación, en el trabajo de campo o dar cuenta de una investigación finalizada. En el primer caso se procura mostrar los supuestos, ideas y dudas que dan sustento a la propuesta o proyecto de investigación.

Para las personas que están en el trabajo de campo o que han concluido su investigación el objetivo es narrar su proceso, los resultados o datos, desde una óptica crítica y reflexiva.

El Encuentro se estructura por mesas ${ }^{7}$ o paneles donde las y los estudiantes tras presentar

$6 \quad$ La Semana Universitaria se celebra en la semana del 24 de abril, fecha que conmemora la contundente participación del movimiento estudiantil universitario en las luchas en contra de la Aluminium Company of America (ALCOA) en 1970. El Encuentro Nuevas Voces en Ciencias Sociales propone sumarle la nota académica a los ya existentes espacios para las actividades artísticas, culturales y recreativas usuales en esta semana.

7 La mesa está compuesta por ponentes, moderador y comentarista. El moderador explica la forma de trabajo, públicamente su ponencia, pasan a recibir retroalimentación de una persona comentarista que motive el siguiente paso, que consiste en abrir un diálogo con el público.

En sus inicios, para el desarrollo del Encuentro el IIS le hacía la solicitud de participación únicamente a las Escuelas de la Facultad de Ciencias Sociales de la UCR, mismas que proponían a algunos de sus graduandos más sobresalientes. Posteriormente, se fueron generando redes entre los mismos estudiantes participantes, los cuales recomiendan a compañeros con trabajos valiosos.

Estas redes han permitido contar con la participación de jóvenes investigadores e investigadoras de otras Facultades, así como de estudiantes de otras universidades públicas del país, por ejemplo, de la Universidad Nacional (UNA) y de la Universidad Estatal a Distancia (UNED).

Asimismo, como parte de los cambios que se han generado en el desarrollo de los Encuentros, se encuentra la incorporación de estudiantes inscritos en los Proyectos de Iniciativas Estudiantiles, un espacio naciente en el que proyectos de investigación -acción social formulados por estudiantes, reciben apoyo para ejecutarse por parte de la Vicerrectoría de Acción Social de la UCR. La participación de las y los estudiantes que desarrollan sus proyectos de acción social imprime un sello particular a la actividad y plantean una serie de reflexiones críticas sobre la relación entre la investigación, la acción social y los "sujetos" de investigación, así como los límites de las barreras disciplinarias para el desarrollo de la investigación acción-social. La Imagen 1 representa a un productor de papa de la localidad de Tierra Blanca de Cartago, exponiendo sus saberes acerca de este cultivo.

controla el tiempo y dispone la palabra de las personas, el o la comentarista realiza el comentario de los trabajos presentados en la mesa. 


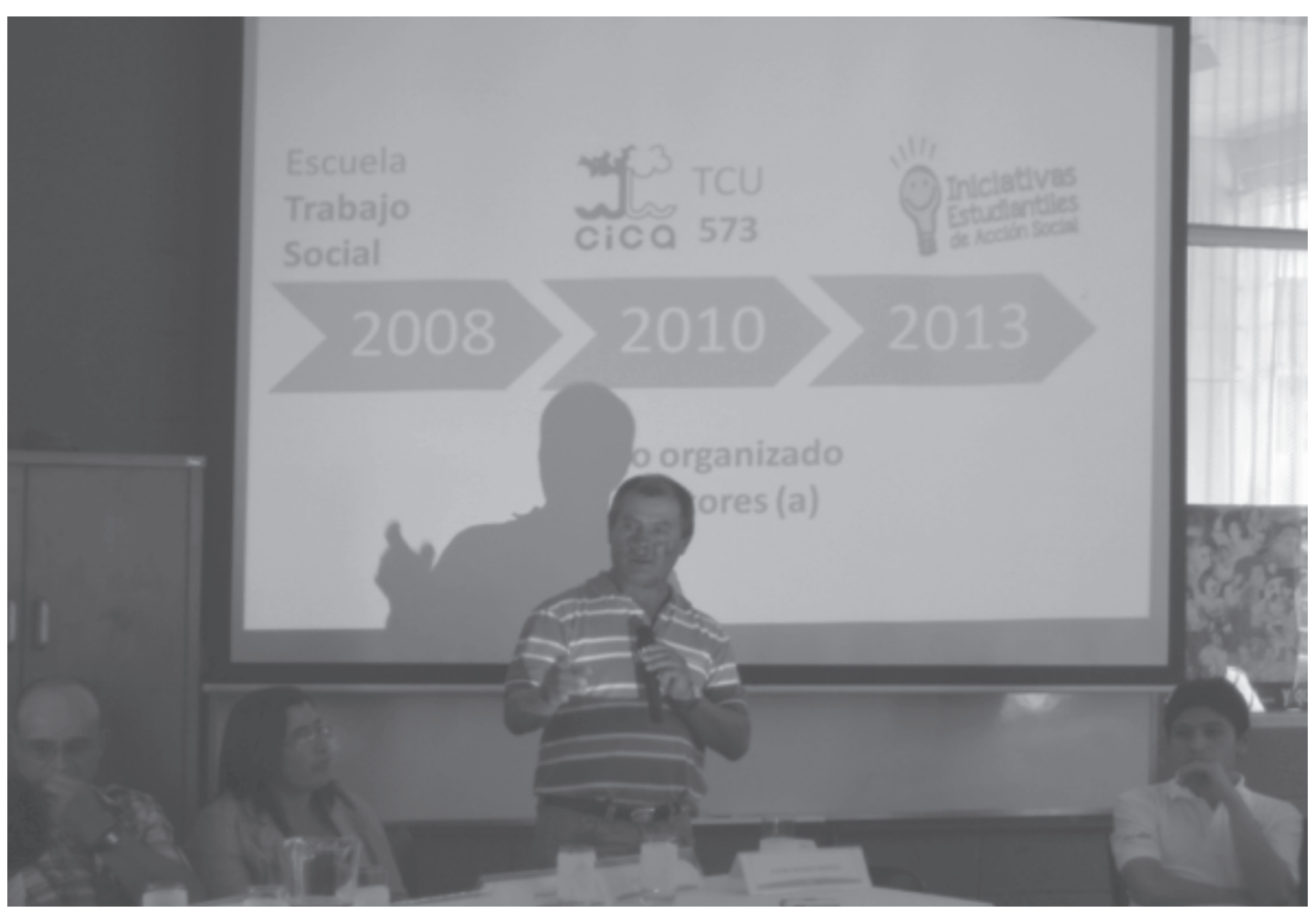

Imagen 1

Mesa Conflicto agrario y procesos organizativos. La transformación es posible Nuevas Voces, 2013 / Fotografía IIS

Además, se ha contado con la participación de estudiantes y jóvenes que investigan y hacen reflexiones críticas sobre la realidad social por su cuenta. Estos dos aspectos han favorecido aún más la creación de redes, la colaboración académica entre jóvenes investigadores y la diversidad en las metodologías, temáticas y reflexiones abordadas en los Encuentros.

Cabe destacar que Nuevas Voces en Ciencias Sociales no sólo son las presentaciones de las ponencias en 2 días de la Semana Universitaria, este se ve compuesto por diversas facetas, en donde se busca garantizar un proceso colectivo con la participación activa de las y los estudiantes investigadores, y participantes de los encuentros anteriores.

De esta manera, Nuevas Voces inicia su proceso con una reunión de balance, donde los participantes evalúan y retroalimentan críticamente la construcción del espacio, y proponen elementos para la producción del siguiente Encuentro. Los participantes de cada año son quienes a partir de la evaluación del anterior se encargan de distintos aspectos del diseño del próximo concurso, por ejemplo, son quienes proponen la batería de temas a priorizar en el cartel del concurso, y a organizar la logística y la comunicación del Encuentro. De manera que cada Nuevas Voces busca ser una experiencia académica distinta, construida horizontalmente combinando las evaluaciones de los anteriores participantes con la creatividad e imaginación de los actuales.

Para el espacio de Nuevas Voces en Ciencias Sociales una de sus principales cualidades son sus redes, por lo que algunas de las y los estudiantes que participaron en los Encuentros, continúan aportando cada año en la elaboración de la actividad, ya sea como coordinadores, comunicadores, en logística o bien, como comentaristas o moderadores el día del encuentro, entre otras. 
La generación de redes de acompañamiento y de intercambio de conocimiento entre las y los participantes de Nuevas Voces, ha significado la participación activa de las y los expositores en las distintas etapas de planificación, desarrollo y evaluación del Encuentro. En relación con lo anterior, una de las participantes del Encuentro NV señala que:

"Rescato la auto gestión en la organización y el compromiso de la gente. Así como la posibilidad de presentar en un espacio alternativo los proyectos o líneas de trabajo".

(Catalina Arguello, Estudiante de Psicología, 2010)

Tal como lo señala la participante un aspecto central es el tema de la "auto gestión" en tanto son las mismas y los mismos estudiantes involucrados, quienes se encargan de la logística y la comunicación de la actividad, apoyados con los recursos humanos y materiales que el Instituto de Investigaciones Sociales, la Facultad de Ciencias Sociales y la Vicerrectoría de Investigación les puedan aportar.

Asimismo, parte de las reflexiones que se plantean en los espacios de reunión y de discusión de Nuevas Voces, es el cuestionamiento a las dinámicas y lógicas hegemónicas en el ámbito académico, por lo cual a partir de la participación activa y colectiva de los y las estudiantes se cuestiona el adulto centrismo y la verticalidad en los eventos académicos, por lo cual cada encuentro de Nuevas Voces en Ciencias Sociales se plantea hacer una ruptura con estas dinámicas (ver Imagen 2).

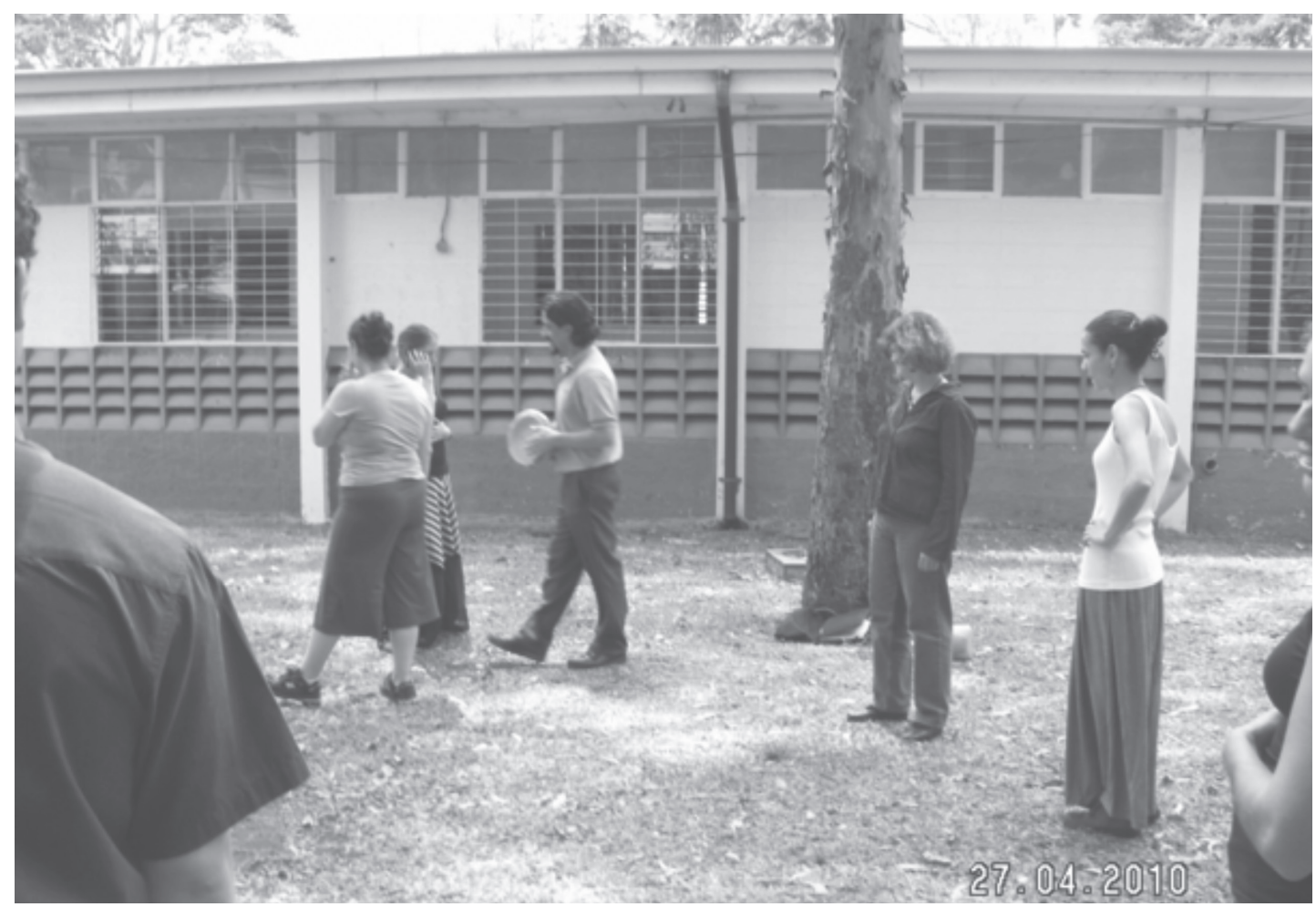

Imagen 2

Nuevas Voces en Ciencias Sociales, 2010

Fotografía IIS 


\section{Nuevas Voces a la luz de la investigación social}

Al ser una actividad que podríamos caracterizar como de iniciación a la investigación, hay participantes para quienes ha constituido una de sus primeras experiencias académicas. Lo anterior se traduce en experiencias heterogéneas. Por ejemplo, a quienes no han defendido aún sus trabajos finales de graduación, NV les permite discutir sus tesis con el público y compañeros, facultando una mejor preparación para el día de la defensa, como nos lo indica la siguiente reflexión:

"Me aportó experiencia para exponer mi proyecto de investigación ahora que estoy cerca de hacer el examen de candidatura. Además las preguntas y observaciones que me hicieron me sirvieron para hacer cambios y foguearme para posibles preguntas que me puedan realizar. Además fui a muchas de las exposiciones de los compañeros por lo que aprendí mucho de diversos temas" (José Luis Rodríguez Fournier, Estudiante de Sociología y Gestión Ambiental, 2010).

De esta manera, Nuevas Voces aporta hacia la presentación exitosa de los Trabajos Finales de Graduación así como, en la continuidad de los estudios. Esto pues una de las principales vocaciones es el acompañamiento que realiza a las personas participantes, en la preparación de las ponencias sobre sus estudios.

Además se generan redes académicas y de apoyo interdisciplinario, que permiten la construcción de comunidades con la cual discutir intereses afines, perspectivas diversas y ampliar los horizontes de conocimiento, al compartir inquietudes y preguntas de investigación, metodologías y enfoques, tal como reflexiona una de las participantes:

\footnotetext{
"Me ha permitido reflexionar e integrar líneas de investigación, compartir con otras personas nuestro trabajo y ver el desarrollo de temas novedosos por gente joven" (Catalina Arguello, Estudiante de Psicología, 2010).
}

La discusión se enriquece cuando participan personas de distintas generaciones, pues permite crear un intercambio de consejos y observaciones basadas en la experiencia, mientras otros aportan nuevos temas y perspectivas de investigación, o en palabras de María Alejandra:
"En buena hora existe Nuevas Voces. Voy a empezar por decir eso. Creo que es un buen espacio para poner en común y discutir lo que estamos investigando. En una buena ventana, no solo con profesionales de mayor experiencia, sino también entre compañeros(as) "contemporáneos". En mi caso, fue una experiencia que me ayudó como entrenamiento para lo que será la defensa de mi tesis y creo que eso tiene gran valor" (Ma. Alejandra Escalona, Estudiantes de Ciencias Políticas, 2010)

Por otra parte, a través de las distintas presentaciones de $\mathrm{NV}$, hemos identificado cómo sus ponentes aportan año con año, nuevos acercamientos a las diferentes problemáticas que investigan. Algunos énfasis que hemos notado a través de los encuentros son: el análisis crítico de discursos y de prácticas de psicopatologización (Sánchez, 2008; Soto, 2013 y Bolaños, 2013); un retorno a los temas de lo agrario y el mundo rural (Martínez y Morales; Quesada; Guillén; y Solera y Muñoz; 2013) así como, propuestas novedosas en el estudio de geografías de poder en los espacios urbanos y rurales en el marco del neoliberalismo económico (Navarro, 2013 y Rojas, 2013).

Para ejemplificar sobre el tipo de trabajos de investigación expuestos en los encuentros de Nuevas Voces, citamos el TFG de Santiago Navarro (2012), el cual presentó un estudio de caso que aborda el impacto del Hotel Riu en la comunidad de Matapalo, Guanacaste. Navarro realizó en su tesis un acercamiento etnográfico, trasladándose a la zona para entrevistar y convivir por un tiempo con los trabajadores de la construcción y el servicio turístico. Además, como muestra de su estrecho compromiso con la comunidad en la que realizó su trabajo, Navarro le dedica su tesis al trabajador: "Rafael Antonio Pérez Sánchez, trabajador nicaragüense muerto impunemente en el 2008 por las condiciones de explotación del hotel Riu y el sistema turístico transnacional" (Navarro, 2013: 3).

Asimismo, en el VIII Encuentro de NV se desarrollaron temas vinculados con la ruralidad y el conflicto agrario en Costa Rica. Las y los ponentes señalan a este espacio como una puerta para posicionar el tema agrario en las ciencias sociales, debido a que este ha sido un tema relegado desde hace varias décadas. Además, las y los participantes señalan que utilizaron una serie de metodologías y formas de acercamiento con las 
comunidades rurales innovadoras, que les permitieron ir más allá de las técnicas y metodologías utilizadas tradicionalmente según las carreras que cursan.

Además, para algunas y algunos participantes NV este espacio ha constituido un paso en su carrera como docentes e investigadores, por ejemplo vehiculizando la publicación de artículos científicos y memorias que influyen en su quehacer y dando a conocer sus investigaciones. Muchos de los y las ponentes, han publicado en revistas científicas, artículos con base en sus ponencias. Asimismo, se ha apoyado para la publicación de cinco artículos elaborados por participantes del III Encuentro de Nuevas Voces en Ciencias Sociales, el Anuario de Estudios Centroamericanos N $^{\circ} 33-34$, 2007-2008 reúne.

A más de de discutir problemas de la realidad nacional a través de la presentación de TFG's, hemos abierto mesas para la discusión de temas pertinentes a la juventud universitaria, por ejemplo, las condiciones laborales en el interinazgo dentro de la UCR se abordaron en el 2009 con la ponencia Pulsearla, tensiones y empuje, a cargo de varias y varios jóvenes investigadores del IIS, y en el 2010, Alberto Sánchez, Gabriela Brenes y Priscilla Carballo discutieron los Desafios del trabajo joven en las sedes universitarias.

Ahora bien, respecto a los esfuerzos que se realiza para la difusión y presentación de resultados, podemos decir que se ha apoyado en diversos aspectos, tales como: la escritura, presentación oral y audiovisual de las ponencias; la escritura de artículos científicos. En síntesis, este proceso es académico, colectivo y subjetivo, pues involucra trabajar en conjunto y desde la diversidad, con las ansiedades que despiertan los procesos de investigación.

Asimismo, se ha impulsado la difusión y devolución de las investigaciones en medios de comunicación alternativos. De manera que el Encuentro ha logrado posicionarse en los medios de comunicación internos y externos a la Universidad. Esto nos permite trascender el espacio habitual del campus universitario Rodrigo Facio, construir espacios de intercambio de experiencias de investigación, y abrir el diálogo con las comunidades. Sigue pendiente digitalizar en mayor medida nuestra presencia en los medios y también, debe seguir potenciándose la comunicación personalizada.

Una de las principales expresiones de las relaciones con lo cotidiano, se vive con las comunidades con las cuáles investigamos o intervenimos. Una fase central en ese proceso se concreta en la devolución y en dar cuenta a las comunidades de los resultados y retroalimentación sobre el proceso mutuo. La elaboración que esto conlleva debería permitir formular precisiones y discusiones relacionadas con los contextos sobre los cuales intervenimos.

Dentro de nuevas voces se ha abierto espacios de discusión horizontal, que permiten a las mismas personas que fueron "sujeto" de investigación, participar, no sólo como público, sino también como expositores, rompiendo las lógicas de la investigación social tradicional, desvinculando de la posición de saber y de imposición de verdades a quien investiga, y permitiendo la construcción de conocimientos y aprendizajes mediante el intercambio de saberes.

Por esta razón, también la conformación del público ha sido muy diversa, y ha trascendido el público tradicionalmente universitario, pudiendo contar con la presencia de amas de casa, mujeres pensionadas, personas trans, campesinas y campesinos así como de grupos de la tercera edad. Además, la presencia de públicos distintos a los universitarios, nos exige romper con las lógicas tradicionales académicas de competencia y descalificación proponiendo un diálogo respetuoso e inclusivo entre diversos actores. Esto hace justicia a los propósitos iniciales con los que se creó la actividad:

"Este Encuentro encuentra eco en la idea de abrir las ciencias sociales. Queremos contribuir a que el debate de las ciencias sociales sea más diverso, que haya más voces y con ello ampliar las agendas de investigación. Las ciencias sociales tendrán vigencia en la medida en que recojan y exploren las inquietudes, las demandas de la época.” (Sandoval, 2007).

A manera de cierre, queremos concretar la idea de que a través de NV se suma un esfuerzo más por permitir diálogos diversos: inter generacionales, inter disciplinarios y académicocomunitarios. La participación activa y colectiva en la elaboración del encuentro es fundamental para ello, se trata de un proceso colectivo entre 
y para estudiantes, que permite la construcción de saberes en diálogo, y no en solitario. De esta manera, NV ha aportado a la vida académica de las y los estudiantes, además e rescatar el diálogo y participación de las comunidades.

\section{Conclusiones}

El Encuentro Nuevas Voces en Ciencias Sociales fomenta encuentros estudiantiles que retroalimenten el proceso de investigación a las y los estudiantes en ciencias sociales. Constituye un espacio dirigido a que las y los proponentes para licenciatura y maestría en Ciencias Sociales elaboren o desarrollen redes de discusión académica que permita el enriquecimiento de sus proyectos, y permite intercambios entre estudiantes de la Facultad y afines, con el personal de investigación del Instituto de Investigaciones Sociales.

En este sentido, el Encuentro Nuevas Voces en Ciencias Sociales se ha ido consolidando con el tiempo como una herramienta de encuentro, intercambio, discusión crítica y generación de redes académicas entre estudiantes y jóvenes investigadores. En donde surgen espacios y discusiones necesarias que contribuyen al desarrollo de una investigación social ética y comprometida con los grupos sociales marginados y excluidos; generando así un aporte a la discusión colectiva, creativa y reflexiva sobre el quehacer y el devenir de las investigación en ciencias sociales.

No obstante, está pendiente el reto de continuar avanzando en la comunicación con la Facultad, pues debe darse a conocerse más y mejor el espacio y volverse cada día más accesible al estudiantado.

La enseñanza y producción de las ciencias sociales se enriquece cuando los estudiantes obtienen herramientas para investigar desde diversos enfoques, con multiplicidad de metodologías, así como, haciendo uso de la creatividad, a través de acercamientos sensibles, críticos y transformadores. Esto se traduce en: 1) capacidad para formular preguntas y problemas acordes a exigencias coyunturales y de larga duración; 2) la fortaleza para distanciarse de las agendas impuestas por los discursos hegemónicos potenciados por los grandes organismos internacionales, guiados por los intereses del capital, visibilizando y desmitificando aquellas verdades impuestas que buscan legitimar y reproducir las relaciones de desigualdad y la explotación. Y 3) la habilidad de proponer espacios y propuestas alternativas, críticas, de transformación y ruptura.

Algunos de los retos pendientes que trascienden pero atraviesan tanto al Encuentro como el quehacer diario de las ciencias sociales, son los campos y rencillas de poder disciplinares, las grandes sombrillas teóricas y recetas metodológicas, así como, que se requiere de mayores libertades para desarrollar nuevas temáticas y experimentar con metodologías novedosas, en espacios capaces de fortalecer, acompañar e impulsar procesos de investigación comprometida, en vías de romper con las cadenas disciplinarias y cuestionar los estereotipos de las carreras.

Dado que estamos en un espacio universitario guiado por las vocaciones de la investigación, la docencia y la acción social, debemos subrayar que nunca debe perderse de vista al estudiantado como parte fundamental y activa de las dinámicas que ocurren en la comunidad universitaria. Por esta razón es necesario reconocer el aporte y las reflexiones y críticas o propuestas de éstos para la construcción de un diálogo de saberes y el reconocimiento de sus aportes en la producción de conocimientos por parte de las ciencias sociales. Además, debemos encontrar estrategias que permitan sistematizar y dar continuidad a los proyectos que generan.

\section{Referencia bibliográfica}

Alvarado Alcázar, Alejandro y Martínez Sánchez, Gloriana (2013). "En el tiempo del libre mercado: conflictos y protesta social en Costa Rica durante el año 2012”. En: Revista OSAL Año XIV, $N^{\circ} 33$. (Buenos Aires: CLACSO)

Bolaños, Eduardo (2013) "Psicofármacos, escuela e infancia: Discursos y experiencias de niños/as diagnosticados/as con hiperactividad". Ponencia presentada en el VIII Encuentro Nuevas Voces en Ciencias Sociales (Instituto de Investigaciones Sociales, Universidad de Costa Rica).

Guillén, María José (2013) "Colonialidad del poder en la implantación bananera: 
Precedentes históricos del conflicto agrario en Bambuzal de Sarapiquí". Ponencia presentada en el VIII Encuentro Nuevas Voces en Ciencias Sociales (Instituto de Investigaciones Sociales, Universidad de Costa Rica).

Martínez, Gloriana y Morales, María Paula (2013) "La organización y resistencia de las mujeres rurales: entre la cooptación estatal y los procesos de construcción de autonomía". Ponencia presentada en el VIII Encuentro Nuevas Voces en Ciencias Sociales (Instituto de Investigaciones Sociales, Universidad de Costa Rica).

Mora, Sindy; Paniagua, Laura; Sánchez, Adriana y Zúñiga, Mario (2009) "Pulsearla: tensiones, empuje y creación en el espacio laboral universitario". Ponencia presentada en el IV Encuentro Nuevas Voces en Ciencias Sociales (Instituto de Investigaciones Sociales, Universidad de Costa Rica).

Martín-Baró, Ignacio. "El estudiantado y la estructura universitaria", ECA, $\mathrm{N}^{\circ} 30$, 1975, pp. 638-651.

Navarro, Santiago (2012) "Poder, colonialidad y resistencia en el turismo. La construcción de la periferia del placer en Guanacaste". Ponencia presentada en el VIII Encuentro Nuevas Voces en Ciencias Sociales (Instituto de Investigaciones Sociales, Universidad de Costa Rica).

Quesada; Jorge (2013) "De nuevo la cuestión agraria: resistencia campesina en la Zona Sur". Ponencia presentada en el VIII Encuentro Nuevas Voces en Ciencias Sociales (Instituto de Investigaciones Sociales, Universidad de Costa Rica).

Rojas, Mariana (2012) Entre Avenida 5 y Calle 8. Percepciones y prácticas de las mujeres trabajadores del sexo en San José, Costa Rica. Ponencia presentada en el VII Encuentro Nuevas Voces en Ciencias Sociales (Instituto de Investigaciones Sociales, Universidad de Costa Rica).
Rodríguez Alvarado, Raquel (2008). Cifras relevantes de la educación superior 20002008. Oficina de Planificación de la Educación Superior. Consejo Nacional de Rectores. (San José: Costa Rica).

Sánchez, Adriana (2008) "Locura psicopatologías y sus relaciones con la criminalidad". Ponencia presentada en el III Encuentro Nuevas Voces en Ciencias Sociales (Instituto de Investigaciones Sociales, Universidad de Costa Rica).

Sánchez, Alberto; Brenes, Gabriela y Carballo, Priscilla (2010) "Desafíos del trabajo joven en las sedes universitarias". Ponencia presentada en el V Encuentro Nuevas Voces en Ciencias Sociales (Instituto de Investigaciones Sociales, Universidad de Costa Rica).

Sandoval, Carlos "Discurso de inauguración", II Encuentro Nuevas Voces en Ciencias Sociales, 2007 (Instituto de Investigaciones Sociales, Universidad de Costa Rica).

Solera, Laura y Muñoz, Daniela (2013) "La metáfora de la papa cele: el caso del Servicio de Información Agrícola de Tierra Blanca (SIT) como herramienta de fortalecimiento organizativo de los agricultores y agricultoras de la zona". Ponencia presentada en el VIII Encuentro Nuevas Voces en Ciencias Sociales (Instituto de Investigaciones Sociales, Universidad de Costa Rica).

Soto, Mario (2013) "La (des) patologización de la transexualidad: una discusión sobre el abordaje psiquiátrico de las identidades de género". Ponencia presentada en el VIII Encuentro Nuevas Voces en Ciencias Sociales (Instituto de Investigaciones Sociales, Universidad de Costa Rica).

Sousa Santos, Boaventura (2007). La universidad en el siglo XXI. Para una reforma democrática y emancipadora de la universidad. En: Revista Umbrales. N.15. CIDES. Universidad Mayor de San Andrés. Argentina. 